\title{
Human-Machine Interface: Design Principles of Interactive Waiting in Web and App
}

\author{
Minghui $\mathrm{Hu}$ \\ School of Mechanical and Vehicular Engineering \\ Beijing Institute of Technology \\ Beijing, China \\ 184639923@qq.com
}

\author{
Peng Bian \\ School of Art and Design \\ North China University of Technology \\ Beijing, China \\ camus10047@163.com
}

\begin{abstract}
This paper analyzed and generalized the content and characteristics of interactive waiting, which should care more about user's psychological sense about waiting time. Based on the discussion, the article advanced several principles to help designer to do user-centered interaction design, such as put user centered and transferred user's waiting attention. By the principle, designer can give a more happy waiting experience to the user. This paper also recapitulated that the perfect interaction makes internet not only a tool, but a friendly partner by caring user experience more.
\end{abstract}

Keywords-interactive design; waiting; web; app

\section{INTRODUCTION}

With the development of internet in china, internet has been integrated into the daily life of the people, such as shopping, playing, learning and working on internet. The scale of China's Internet users had reached 564 million, Internet penetration rate of $42.1 \%$ (2012 Statistics China Internet network development report). It was reported, by January 15, 2013, that many businesses have to break the older business model, and develop network channels to seek new growth point of sales, after affecting by the consumer's attention, attitudes change and other factors in network marketing. [1] By the end of June 2013, the number of website was 2.94 million, grew 260,000 in six months, an increase of 9.6\%. [2]

There are more and more websites were published on the internet and more commodity and information were uploaded to the different pages and sites. So much information obviously exceeds the cognitive abilities of people, and users may feel their energy was used up by complex information when they want to find some interested content from a messy huge warehouse. There is more and more loading page pop up on the screen when the web system serves for user to load data or switch to pages.

\section{Loading ....please wait}

Fig. 1. Example of interactive waiting.
Today, when surfing on the internet to find what we want, we often encounter waiting situations. Waiting for the response of system is very common, when we are installing, starting, switching, and transmitting. Wait is often accompanied by anxiety, complaining, anger, and even despair, and other negative emotions, those kind of emotional experience is very unfavorable, will bring more churn. In the practice, the design of interactive waiting had several obvious disadvantages:

\section{A. Invisible for Users}

A lot of design is not obvious for users, and the text is very small and the color is thin, so users can hardly notice their presence from the background. In another, the designer had forgotten to tell us how the system is running, why the interface did not advance for a long time, and how long the work would be well. Those unintuitive designs derived from the lack of carefully analyzed logic, structure, hierarchy, so users can not understand the mechanism behind the page.

\section{B. No Response to Users,}

When the interactive waiting appears, all information is static: the progress bar does not move ahead, there is no indication of the current progress of the scan. There is no change in the number of this kind of waiting make users instantly generate anxiety and unrest, and they wonder, "when will the loading is complete?", "Is the computer in a normal job?". See the following screenshot: what these ring represent? Did it represent you had or you will well? Why does not show the percent?

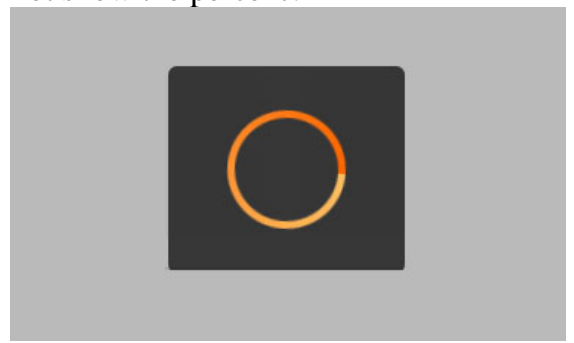

Fig. 2. Example of interactive waiting. 


\section{Can't Stop the Process}

User-friendly interactive waiting should be easily identified and understood for users, it is the basic principle for designer. While there was another case that the process of waiting cannot be stopped by people as will and people must stood still to wait it finish.

\section{DISCUSSED CHARACTERISTICS}

From the perspective of user experience, interactive waiting is a kind of technology which cares about how to make the web easy, efficient and pleasant enough to use for people. So designer should understand the target users' expectations, behavior and the "human" itself psychological, before they put out their demo.

\section{A. Psychoanalysis}

1) People may feel idle waiting for something to do longer than having something to do;

2) When wait for a long time, user will becomes anxiety, anger, which may make waiting seem longer than the actual, so the designer should calm down the user by some special treatment;

3) People may feel indefinite waiting time longer than wait limited;

\section{B. Uniqueness}

Generally, the designer of interactive waiting may has only one copy of program running on line which can be used in different process for all users. In order to make web system easy to use for all visitors to finish their task, the activities of design should be based on the research of user's psychology and behavior, and be combined with local humanism and preference to make the project suite for most of people.

\section{Participation}

The internet is an interactive tool for today's people, by which people can do a lot of interactivity. People can interact with viewers in enjoyable experience and achieve better effect to finish works. The interactive waiting is not only help visitor interact with website quickly and efficiently, but also help to take the control of waiting.

\section{Understandable}

The design of web and app must be easy to read, understand and use, so the interactive manner of system should be clear for user to operate, not need to undertake special training, so do the interactive waiting. Designer must make interface and interaction more visible and understandable to enable the contents to be correctly interpreted. [3] Designer should guarantee the information, function and framework to be distinct and easy to be distinguished.

\section{DESIGN PRINCIPLES}

During the process of interactive design, we should think and design from a user perspective. The design should set a target aim to let users easily find the target, understand the logical, easily interact with system, and feedback to user's action.

\section{A. User-Centered Principle}

Whether can let users rapidly obtain their needed, is primary standard to measure interaction's efficiency. [4] Efficiency of Information interaction with users to a great extent is determined by the method of interaction design, and the logic organization for users. The design should understand what user want, and tell them where he is, what he can do. So the users can quickly catch the meaning and switch to their destination.

In order to finish work by web, people need to read text on the page, understand the business logical to find their target, and then select their want, and click the links to next step. Designer must fully comprehend the psychological needs of audience and the rules of information cognition to make users more comfortable to enjoy the services provided by web system.

\section{B. Transferred User's Attention}

The common practice to reduce waiting time is the transfer of user's attention away which may generate negative emotions. Allowing users to have other things to do is very practical in real life. When users open a recommended application, designer can provide users a funny little piece, and allow users to read through little piece to pass the time, unlike a general application telling the user data in the buffer. In this way, people can have their spare time well. Designer can also provide cartoon animation to distract user's attention when slide out more free APP.

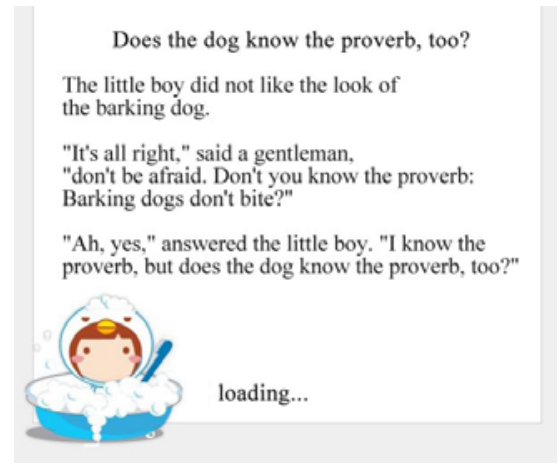

Fig. 3. Example of funny piece.

\section{Tell User Waiting Time}

The design must be clear for users. [5] The reason why our user was anxiety, anger and other negative emotions, is not just because of the length of waiting time, in fact, most important reason is to wait for the results while unknown the end. So an effective method to reduce user's anxiety is to let users know the duration of the whole process of waiting and the current wait time remaining. For example, windows combine time tips and the progress bar to remind people the copy process. 


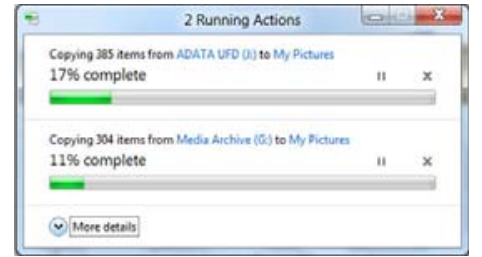

Fig. 4. The process of copy.

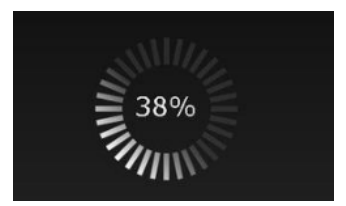

Fig. 5. Example of waiting for loading.

\section{Preview}

Allowing users to preview the contents of waiting, in fact, is a kind of asymptotic way to display the contents and a method to let users understand the content of the framework to comfort agitated mood when the user waits. This type of treatment presented word in the title firstly, then the content can be slowly buffered; for information on the picture is the first low-quality pictures to show the user image contours, and then again presents user high-quality pictures.

APP Store used the asymptotic formula in this way. When people open the APP Store in the classification page, labels first appeared in the title and the content is still being buffered, and when you read the title, contents followed almost appeared.

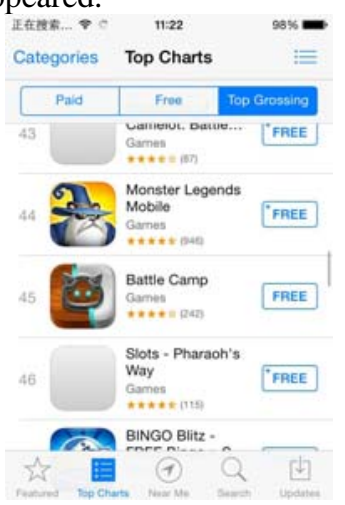

Fig. 6. Example of waiting in app store.

\section{E. Do more for user}

In order to help users to finish their work, the designer should tell the current place to them, so they can know where they is, where they had been and where they can go. That means to allow users to set the program to do something automatically after the end of waiting, such as help people to the next step or shutdown automatically, etc. With this feature, users can no longer keep in front of the machine bored waiting.

\section{F. Response to User}

When there is a very short waiting time, it is necessary to provide a significant progress indicator or the remaining time notification. In order to improve the experience of user, it is still necessary to show buffer identification, such as the Windows operating system buffer hourglass small logo.

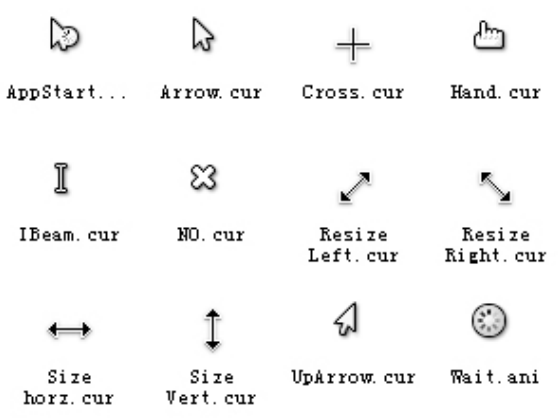

\section{Fig. 7. Example of arrow}

\section{CONCLUSION}

The design of interactive waiting should pay more attention to user's psychology and manner to do a great visual design and interaction design for users. In order to avoid people to feel the waiting bored, interactive waiting should distract people's attention and calm down their feeling by let them funny to do something.

The study of interaction design plays a decisive role in the human-machine interface, predominate the development of web application. The clarity, readability, maneuverability and convenience of the human-machine interface require designer to carry out their design with the guide of human concept and the foundation of cognitive psychology, which will lead to the consumer-centered, reassuring, comfortable and consumer-satisfied humanmachine interface.

\section{FORECAST}

Taking people-oriented design as a guide, innovate a way of interaction design of web, by which the users will be satisfied with what they used, and be interested in their business, functions, and meet the internal needs of the consumers.

\section{ACKNOWLEDGMENT}

The author will acknowledge the unbreakable enthusiasm of the participants of this study. We also wish to convey our thanks for the valuable suggestions provided from reviewers.

\section{REFERENCES}

[1] 2012 Statistical Report on Internet Development in China.

[2] 2013 Statistical Report on Internet Development in China.

[3] Alan Cooper, Robert Riemann, About face 3: The essentials of Interaction Design, China: Electronics Industry, 2012 
[4] Donald A. Norman, the Design of Everyday Things, China: CITIC, 2003.
[5] Alan Cooper, The Way of Running Interaction Design, China: Electronics Industry, 2012 